\title{
Biophotonics in Bioengineering
}

Biophotonics encompasses the fundamental interactions of light and biological materials. The field has produced optical imaging, diagnostic and therapeutic devices that have impacted modern medicine. It has provided major advances in biomedical research through development of technologies for high resolution microscopy, and contributed to the progress in molecular labelling through the development of fluorescent proteins, and medically relevant contrast and therapeutic agents. Many of these interdisciplinary activities have involved bioengineers and have grown out of bioengineering departments. This special issue of ABME encompasses research papers at the interface of biophotonics and bioengineering which we have solicited from internationally renowned researchers. This includes a total of 16 review articles to introduce the field to a general audience of bioengineers, and the topics have been selected to cover a broad range of biophotonics research areas.

The subjects of endoscopy and tissue optics are covered in reviews by Stoyanov on how computational techniques are being used in surgical vision [Stoyanov, D "Surgical Vision" DOI: 10.1007/s10439-011-0441-z], and Fantini and Sassaroli who detail the current state of nearinfrared optical mammography for breast cancer detection [Fantini S, Sassaroli A "Near-Infrared Optical Mammography for Breast Cancer Detection with Intrinsic Contrast" DOI: 10.1007/s10439-011-0404-4]. The promising diagnostic role of optical spectroscopic techniques are described by Marcu [Marcu, L "Fluorescence Lifetime Techniques in Medical Applications"], who has reviewed the applications of fluorescence lifetime imaging for the diagnosis of different tissue pathologies using only naturally occurring fluorophores.

Reviews of how ultrasound and optics may be combined for enhanced imaging are presented by Murray et al. who describe the monitoring of therapeutic responses with acousto-optic imaging [Murray TW, Lai P, Roy RA "Measuring tissue properties and monitoring therapeutic responses using acousto-optic imaging" DOI: $10.1007 / \mathrm{s} 10439-011-0425-z]$, and Sarantopoulos et al. appraise the use of opto-acoustic methods in interventional imaging [Sarantopoulos A, Beziere N, Ntziachristos V. "Optical and Opto-Acoustic Interventional Imaging" DOI: 10.1007/s10439-011-0501-4]. Microscopic methods for imaging cellular and subcellular processes from clinical and research perspectives are covered in papers by Miao et al. focusing on optical projection tomographic microscopy, and Perry et al. on the use of two photon and second harmonic microscopy [Miao Q, Reeves AP, Patten FW, Seibel EJ “Multimodal 3D Imaging of Cells and Tissue, Bridging the Gap Between Clinical and Research Microscopy" DOI: 10.1007/s10439-011-0411-5].

Finally, we have selected papers on photo- and thermal-therapy that introduce mechanisms through which light may be used for tissue therapy. Chung et al. describe techniques in low-level laser therapy and how they are being applied to different diseases and tissues [Chung $\mathrm{H}$, Dai T, Sharma SK, Huang YY, Carroll JD, Hamblin MR "The Nuts and Bolts of Low-level Laser (Light) Therapy" DOI: 10.1007/s10439-011-0454-7]. Chit Yaw et al. examine the use of photosensitizers for photodynamic diagnosis, and the combination of photothermal and photodynamic therapies [Olivo M, Fu CY, Raghavan V, Lau WK. "New Frontier in Hypericin-Mediated Diagnosis of Cancer with Current Optical Technologies" DOI: 10.1007/s10439-011-0462-7] .

We hope that the papers selected will be of interest to the bioengineering community in general, and thank the investigators for their outstanding contributions.

DANIEL ELSON

Imperial College London

Electronic mail: daniel.elson@imperial.ac.uk

Bahman Anvari

University of California 